\title{
Impact of long-axis function on cardiac surgical outcomes in patients with radiation-associated heart disease
}

\author{
Srisakul Chirakarnjanakorn, MD, ${ }^{\mathrm{a}}$ Zoran B. Popović, MD, PhD, ${ }^{\mathrm{a}}$ Willis Wu, MD, ${ }^{\mathrm{a}}$ Ahmad Masri, MD, \\ Nicholas G. Smedira, MD, ${ }^{b}$ Bruce W. Lytle, MD, ${ }^{b}$ Brian P. Griffin, MD, ${ }^{a}$ and Milind Y. Desai, MD ${ }^{a}$
}

\begin{abstract}
Background: Malignancy-associated thoracic radiation leads to radiationassociated cardiac disease (RACD) that often necessitates cardiac surgery. Myocardial dysfunction is common in patients with RACD. We sought to determine the predictive value of global left ventricular ejection fraction and long-axis function left ventricular global longitudinal strain (LV-GLS) in such patients.
\end{abstract}

Methods: We studied 163 patients (age, $63 \pm 14$ years; 74\% women) who had RACD and underwent cardiac surgery (20\% had reoperations) between 2000 and 2003. In addition to standard echocardiography, LV-GLS (\%) was derived from the average of 18 segments in 3 apical views of the left ventricle, using velocity vector imaging. Standard clinical and demographic parameters were recorded. All-cause mortality was recorded.

Results: The mean duration between cardiac surgery and the last chest radiation was $18 \pm 12$ years. The median European System for Cardiac Operative Risk Evaluation (EuroSCORE) was 8, and 88 patients died over $6.6 \pm 4$ years. A total of $52 \%$ of patients had $\geq \mathrm{II}+$ mitral regurgitation; $23 \%$ of patients had severe aortic stenosis; and $39 \%$ of patients had $\geq \mathrm{II}+$ tricuspid regurgitation. The mean left ventricular ejection fraction was $54 \% \pm 13 \%$, and the mean LV-GLS was $-12.9 \% \pm 4 \%$. In a Cox proportional survival analysis, lower LV-GLS was predictive of mortality in univariable analysis (hazard ratio, 1.07 (95\% confidence interval, 1.01-1.14); $P=.006$ ); however, after adjustment for other variables, the association became nonsignificant. In patients with a EuroSCORE $<$ median, abnormal LV-GLS $(<-14.5 \%)$ was associated with significantly higher mortality (48\%), compared with those with normal LV-GLS $(32 \%)$.

Conclusions: In patients who have RACD and undergo cardiac surgery, LV-GLS does not sufficiently discriminate and is not independently predictive of long-term outcomes. However, in patients with a low EuroSCORE, abnormal LV-GLS was associated with higher mortality, compared with those with normal LV-GLS. (J Thorac Cardiovasc Surg 2015;149:1643-51)

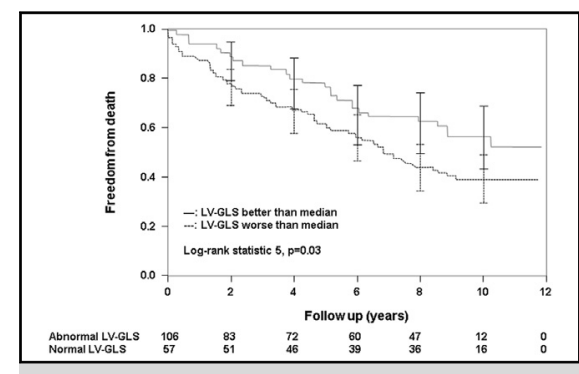

Kaplan-Meier survival curves of the entire study population, separated on the basis of preserved versus abnormal left ventricular global longitudinal strain $(L V-G L S)$

\section{Central Message}

In 163 patients with radiation-associated cardiac disease, lower left ventricular global longitudinal strain (LV-GLS) was predictive of mortality on univariable (HR, 1.07 [1.01-1.14]; $P=.006$ ) but not on multivariable analysis. In patients with low Euroscore, LV-GLS $<-14.5 \%$ was associated with significantly higher mortality (48\%) versus higher LV-GLS (32\%).

\section{Perspective}

Radiation-associated cardiac disease (RACD) patients are a challenging group who have a heterogeneous presentation (coronary artery disease, valvular disease, cardiomyopathy or pericardial disease) often requiring complex cardiac surgeries. There is a high rate of mortality that is not entirely explained by progressive decline in global left ventricular (LV) function Assessment of LV-global longitudinal strain (GLS), while being more sensitive at determining regional $\mathrm{LV}$ dysfunction, does not add independent incremental utility to determine long-term outcomes, in addition to Euroscore. However, patients with preserved LV ejection fraction but abnormal LVGLS represent a higher risk subgroup in which assessment of LV mechanics might provide incremental utility. Similarly, lower risk patients with a low Euroscore but abnormal LV GLS represent a higher risk subgroup in which assessment of LV mechanics might provide incremental utility. In RACD patients, a comprehensive cardiopulmonary evaluation is necessary before referring them for cardiac surgery. In patients that show high-risk features, alternate therapies like percutaneous coronary intervention and transcatheter valvular therapies should be considered.

See Editorial Commentary page 1651.

\footnotetext{
From the Departments of Cardiovascular Medicine ${ }^{\mathrm{a}}$ and Cardiothoracic Surgery, ${ }^{\mathrm{b}}$ Heart and Vascular Institute, Cleveland Clinic, Cleveland, Ohio.

Received for publication Aug 10, 2014; revisions received Jan 19, 2015; accepted for publication Jan 29, 2015; available ahead of print March 6, 2015
}

\footnotetext{
Address for reprints: Milind Y. Desai, MD, Department of Cardiovascular Medicine, Heart and Vascular Institute, Cleveland Clinic, 9500 Euclid Ave, Desk J1-5, Cleveland, OH 44195 (E-mail: desaim2@ccf.org). $0022-5223 / \$ 36.00$

Copyright () 2015 by The American Association for Thoracic Surgery

http://dx.doi.org/10.1016/j.jtcvs.2015.01.045
} 


$\begin{array}{ll}\text { Abbreviations and Acronyms } \\ \text { CABG } & =\text { coronary artery bypass grafting } \\ \text { CI } & =\text { confidence interval } \\ \text { EuroSCORE } & =\text { European System for Cardiac } \\ & \text { Operative Risk Evaluation } \\ \text { GLS } & =\text { global longitudinal strain } \\ \text { HR } & =\text { hazard ratio } \\ \text { LV } & =\text { left ventricular } \\ \text { LVEF } & =\text { left ventricular ejection fraction } \\ \text { RACD } & =\text { radiation-associated cardiac disease }\end{array}$

Supplemental material is available online.

Radiation therapy is an effective treatment for many kinds of malignancies involving the mediastinum and thorax. However, over the past 30 years, the long-term deleterious effects of therapeutic radiation on the cardiovascular system have been increasingly recognized. Cardiovascular issues are now the leading causes of death in survivors of those malignancies. ${ }^{1-6}$

Radiation-associated cardiac disease (RACD) may present in a heterogeneous manner that can be dominated by proximal coronary artery stenoses, valvular lesions, constrictive pericarditis, myocardial disease, conduction abnormalities, or their combination. ${ }^{7-11}$ These effects are often insidious, with symptoms occurring years after completion of the original radiation therapy. Given this long latency period before cardiac symptoms become overt, failure to recognize the impact of radiation on cardiac manifestations is common. Thus, the precise prevalence of cardiac morbidity in those who previously received therapeutic thoracic radiation is unknown, and likely depends on radiation dose, use of concomitant chemotherapy, other cardiac comorbidities, and radiation beam direction and shielding. Some studies ${ }^{7,11}$ have suggested that the frequency of myocardial fibrosis is as high as $63 \%$, and that the prevalence of subclinical left ventricular (LV) dysfunction ranges from $39 \%$ to $57 \%$.

Although RACD patients often require cardiovascular surgical intervention, their postoperative morbidity and mortality is much higher than would be expected based on standard risk scores. ${ }^{2,3,5,12-16}$ Although the cause of this discrepancy is likely multifactorial, a possible culprit is myocardial dysfunction. Radiation-associated cardiac disease may lead to myocardial dysfunction by virtue of the effects of radiation itself, which may be compounded by the use of chemotherapeutic agents with adverse cardiac effects in this population and further influenced by the ventricular dysfunction that accompanies decompensated valve disease and coronary artery disease.

Although the LV ejection fraction (EF) and volumes are valuable in estimating risk across populations when there is a wide spectrum of abnormality-from normal to severely abnormal-their prognostic value diminishes when the spectrum of abnormality is narrower. Advanced echocardiographic techniques such as speckle tracking (that enable us to measure LV strain) may be more sensitive in detecting long-axis abnormalities in myocardial mechanics, and may indicate pathology before this is evident on conventional indices of LV function. ${ }^{17-19}$ Given the potential importance of myocardial dysfunction to surgical outcomes in patients with prior radiation exposure, we sought to: (1) determine whether LV global longitudinal strain (LV-GLS) can independently predict long-term mortality in RACD patients undergoing cardiac surgery; and (2) investigate the time-dependent changes of LV size and function after cardiac surgery in this population, by using longitudinal data analysis.

\section{METHODS \\ Study Population}

This was a retrospective observational study of 163 consecutive patients with RACD who underwent cardiothoracic surgery at our center between 2000 and 2003. All patients had a detailed echocardiogram performed before their surgery. From an original study population of 173 such patients, we excluded 10 patients in cases in which high-quality offline LV-GLS analysis could not be performed. All patients had a history of malignancy that required chest irradiation and who subsequently developed coronary and/or valvular disease that required cardiothoracic surgery. The diagnosis of RACD was made after a thorough clinical and echocardiographic evaluation was conducted by experienced cardiologists. In this group, type of prior malignancy and area of radiation were ascertained. When available, the year of the most recent radiation dose was recorded. In addition, the approximate radiation dosages for various malignancies were recorded, based on historic data. All patients were cleared by oncology to undergo a cardiac surgical procedure.

\section{Clinical Data}

All clinical and demographic data were assembled using electronic medical records, after appropriate approval by the institutional review board. History and type of prior cardiac surgery were recorded. A record was made of medications at the time of initial presentation, and whether these were initiated in the postoperative period. Presence of permanent atrial fibrillation, defined according to guidelines, at baseline, and at the time of discharge, was ascertained ${ }^{20}$ Presence of an automated implantable cardioverter defibrillator and need for a permanent pacemaker were recorded.

The details of cardiac surgery were recorded prospectively, and categorized as follows: (1) coronary artery bypass grafting (CABG); (2) $\mathrm{CABG}+1$ valve repair and/or replacement; (3) CABG $+\geq 2$ valve repairs and/or replacements; (4) 1 valve repair and/or replacement; (5) $\geq 2$ valve repairs and/or replacements; (6) others (including pericardiectomy, transplantation, LV assist device, aortic surgery, and myectomy). In patients that underwent $\mathrm{CABG}$, the number of bypassed vessels was recorded. Based on the preoperative data, the additive EuroSCORE (European System for Cardiac Operative Risk Evaluation) was calculated to predict risk of postoperative mortality. ${ }^{21}$ 


\section{Follow-up}

The beginning of follow-up was considered to be the date of cardiac surgery at our institution. Death notification was obtained from the medical record or from the US Social Security Death Index database, and survival was ascertained during follow-up (the most recent query was in March 2011). If the information was available, the cause of death was ascertained. Postoperative stroke was defined as transient or permanent neurologic impairment and disability, owing to vascular causes, including episodes lasting $<24$ hours, which were regarded as transient ischemic attacks.

\section{Echocardiographic Analysis}

All patients underwent comprehensive echocardiograms using commercially available instruments (Philips Medical Systems North America, Inc, Bothell, Wash; General Electric Medical Systems, Milwaukee, Wis; and Siemens Medical Solutions USA, Inc, Malvern, Pa) as part of standard pre-workup and postoperative follow-up for heart surgery. Baseline and every subsequent echocardiogram performed during the follow-up period after cardiac surgery at our institution were systematically reanalyzed for functional and volumetric parameters.

Indexed LV end-diastolic volume, LV end-systolic volume, and LVEF were recalculated from apical-view images using the biplane Simpson's method. ${ }^{22}$ Indexed left atrial volume was recalculated using the area-length method. ${ }^{22}$ Additionally, diastolic function, degree of valvular stenosis, and regurgitation were assessed according to standard guidelines, and recorded prospectively. ${ }^{22-25}$ Degree of mitral, aortic, and tricuspid regurgitation was assessed on a scale of 0 to $\mathrm{IV}+(0=$ none; $\mathrm{I}+=$ mild: $\mathrm{II}+=$ moderate; $\mathrm{III}+=$ moderately severe; and $\mathrm{IV}+=$ severe $)$. Right ventricular systolic pressure was recorded from tricuspid regurgitation velocity and estimated right atrial pressure. Peak and/or mean transvalvular gradients, velocity time integrals across valves/LV outflow tract, and diameter of LV outflow tract were recorded. Aortic valve area was calculated using a continuity equation, and aortic stenosis was graded in standard fashion. A dimensionless index (LV outflow tract velocity time integral/aortic velocity time integral) was calculated. In addition, we calculated a mitral valve splitability score (Wilkins score with a minimum possible score of 0 and a maximum possible score of 12). ${ }^{26}$

Subsequently, on preoperative echocardiograms, offline speckle tracking was prospectively performed, and global longitudinal systolic strain was calculated from an average of 18 segmental strains in longaxis, 2-, 3-, and 4-chamber views of the left ventricle at a frame rate of $>30$ frames/second (Velocity Vector Imaging, Siemens Medical Solutions USA, Inc, Mountain View, Calif). ${ }^{27-30}$ A representative example is shown in Figure E1, $A$ to $D$. In each view, endocardial borders were manually traced in the end-systolic frame, with the software subsequently automatically tracking myocardial deformation. If poor tracking occurred, endocardial borders were readjusted manually until satisfactory tracking was achieved. Strain curves were then generated automatically.

These measurements were made at apical, mid, and basal levels of all visualized LV walls, and composite LV-GLS values for every patient were calculated. The threshold for normal versus abnormal LV-GLS was based on previous data, ${ }^{30}$ which demonstrated a normal value of $-18.6 \%$, and an SD of $1.6 \%$. Assuming a $\pm 99 \%$ confidence interval (CI), we defined abnormal LV-GLS to be a value equal to or lower than $-14.5 \%$. Strain analysis was blinded to clinical and other echocardiographic data.

\section{Statistical Analysis}

Continuous variables were expressed as mean $\pm \mathrm{SD}$, or median. Categoric data are presented as percentage frequency. Differences between groups were compared with the use of the Student $t$ test or ANOVA (for parametric variables) and the Mann-Whitney $U$ test (for nonparametric variables) for continuous variables. A $\chi^{2}$ analysis was used for categoric
TABLE 1. Baseline characteristics of the study population

\begin{tabular}{lc}
\hline \multicolumn{1}{c}{ Characteristic or measure } & Value \\
\hline Age $(\mathrm{y})$ & $63 \pm 14$ \\
Female gender & $121(74)$ \\
Hypertension & $69(40)$ \\
Diabetes Mellitus & $27(16)$ \\
Prior stroke & $18(10)$ \\
Smoking history & $63(36)$ \\
Proximal obstructive CAD & $78(45)$ \\
Prior heart surgery & $34(20)$ \\
ICD & $8(5)$ \\
EuroSCORE & $7.8 \pm 3$ \\
Beta-blockers & $111(64)$ \\
ACE-inhibitors & $65(36)$ \\
Aspirin & $94(54)$ \\
Clopidogrel & $7(4)$ \\
Statins & $56(32)$ \\
Hemoglobin (mg/dl) & $12.8 \pm 2$ \\
Creatinine (mg/dl) & $0.98 \pm 0.5$ \\
Total cholesterol (mg/dl) & $185 \pm 45$ \\
Triglycerides (mg/dl) & $150 \pm 90$ \\
Low-density lipoprotein (mg/dl) & $103 \pm 39$ \\
High-density lipoprotein (mg/dl) & $53 \pm 18$ \\
Type of cardiothoracic surgery & \\
CABG & $24(14)$ \\
CABG +1 valve & $39(23)$ \\
CABG $+\geq 2$ valves & $37(21)$ \\
1 valve only & $38(22)$ \\
O2 valves & $28(16)$ \\
Others & $7(4)$ \\
Number of coronary bypass grafts & $1.2 \pm 1.4$ \\
\hline Values are $n$ \\
Cardiac Oplantable cardiovertor-defibrillator; EuroSCORE, European \\
CABG, coronary artery bypass graft. & System for \\
& \\
&
\end{tabular}

variables. Linear regression between continuous variables was tested using the Pearson correlation coefficient. Cumulative event rates as a function over time were obtained using the Kaplan-Meier product-limit method, and curves of mortality were compared using the log-rank test.

A univariable and multivariable Cox proportional hazards model was developed to determine the independent predictors of mortality. As a first step, all clinically relevant variables, known to be associated with outcomes in this study population, were individually assessed with a univariable analysis. Subsequently, forward, stepwise, multivariable regression analysis was performed using a $P$-value cutoff of $<.1$. The final univariate and multivariate results are represented by hazard ratios (HRs), and their $95 \%$ CIs.

To assess the discriminatory ability of LV-GLS to predict outcomes, we performed receiver operating characteristic analysis, using the data available at the 9-year cutoff, given that all patients had $\geq 9$ years of follow-up. In addition, to assess whether LV-GLS and the probability of untoward outcomes had a linear relationship, we performed the Hosmer-Lemeshow test. In addition, we assessed the time-dependent progression and evolution of systolic LV function (indexed LV volumes and $\mathrm{EF}$ ), and the indexed left-atrial size, after cardiac surgery in individual patients, using a linear mixed-effects model with unstructured covariance for random effects. ${ }^{31}$

First, we assessed the impact of surgery on these echocardiographic parameters. To model the effects of surgery, we used a piecewise linear 
TABLE 2. Baseline echocardiographic data in the study population

\begin{tabular}{lc}
\hline \multicolumn{1}{c}{ Variable } & Value \\
\hline LV ejection fraction $(\%)$ & $54 \pm 14$ \\
LV end-diastolic volume index $\left(\mathrm{mL} / \mathrm{m}^{2}\right)$ & $60 \pm 23$ \\
LV end-systolic volume index $\left(\mathrm{mL} / \mathrm{m}^{2}\right)$ & $30 \pm 20$ \\
Left atrial volume index $\left(\mathrm{mL} / \mathrm{m}^{2}\right)$ & $32 \pm 14$ \\
Diastolic dysfunction, grade & \\
$\quad$ Abnormal relaxation & $83(46)$ \\
$\quad$ Pseudonormal & $21(12)$ \\
$\quad$ Restrictive filling & $19(11)$ \\
Mitral regurgitation grade $\geq 2$ & $85(52)$ \\
Mitral valve splitability score & $4.9 \pm 3$ \\
Aortic regurgitation grade $\geq 2$ & $57(35)$ \\
Dimensionless index for aortic valve & $0.42 \pm 0.2$ \\
Tricuspid regurgitation grade $\geq 2$ & $63(39)$ \\
RVSP (mm Hg) & $41 \pm 15$ \\
LV global longitudinal strain $(\%)$ & $-12.9 \pm 4$ \\
\hline Values are $\mathrm{n}(\%)$, unless otherwise indicated. $L V$, Left ventricular; $R V S P$, right \\
ventricular systolic pressure.
\end{tabular}

regression for both fixed and random effects, with a potential break-point of the 15th, 180th, and 1095th day after cardiac surgery. After that, we assessed possible predictors of the slopes of the individual parameters. For all linear mixed effects, the Akaike information criterion was used to compare the incremental value of the models. The Wald test was used to evaluate the difference in slope of piecewise regression. ${ }^{31}$ Statistical analysis was performed with SPSS version 20.0 (SPSS, Inc, Chicago, Ill).

\section{RESULTS}

\section{Study Population}

The demographic and some baseline echocardiographic characteristics of the patient population are shown in Table 1. No patient had advanced lung disease that was deemed prohibitive for cardiac surgery. All patients had advanced symptoms necessitating cardiac surgery. Nearly half of the population $(45 \%)$ had evidence of obstructive proximal coronary artery disease, including $26(17 \%)$ patients with significant $(\geq 50 \%)$ left main disease. In addition, one fifth of the study population $(\mathrm{n}=34)$ had a history of prior heart surgery. The distribution of prior malignancies and average radiation doses were as follows: $87(53 \%)$ breast (55 gray); 11 (7\%) lung (60 gray); 43 (26\%) Hodgkin's lymphoma (45 gray); 11 (7\%) non-Hodgkin's lymphoma (45 gray); and $11(7 \%)$ others (thymoma, and testicular and thyroid cancers; 45 gray). No patient had a known recurrence of malignancy at the time of cardiac surgery. The duration between current

TABLE 3. Forward, stepwise, multivariable, Cox proportional hazard survival analysis for mortality in the study population

\begin{tabular}{lcc}
\hline \multicolumn{1}{c}{ Factor } & Hazard ratio $(\mathbf{9 5} \% \mathbf{C I})$ & $\boldsymbol{P}$ \\
\hline Beta-blockers & $0.67(0.47-0.96)$ & .04 \\
Aspirin & $0.66(0.44-0.98)$ & .04 \\
EuroSCORE & $1.12(1.04-1.22)$ & .002 \\
\hline
\end{tabular}

CI, Confidence interval; EuroSCORE, European System for Cardiac Operative Risk Evaluation. cardiac surgery and last chest radiation (data available for 96 patients) was $18 \pm 12$ years.

\section{Echocardiographic Characteristics}

The baseline echocardiographic characteristics are shown in Table 2. The distribution of LVEF was as follows: $119(73 \%)$ patients with LVEF $\geq 50 \% ; 36(22 \%)$ with LVEF between $30 \%$ and $50 \%$; and $8(5 \%)$ with LVEF $<30 \%$. A high prevalence of valvular heart disease was found: $85(52 \%)$ patients with at least moderate mitral regurgitation; $10(6 \%)$ with severe mitral stenosis; 57 $(35 \%)$ with at least moderate aortic regurgitation; 38 $(23 \%)$ with severe aortic stenosis; and $63(39 \%)$ with at least moderate tricuspid regurgitation. Two patients had constrictive pericarditis. Significantly elevated right ventricular systolic pressure (>55 mm Hg) was seen in 18 (11\%) patients.

The mean LV-GLS in the study population was $-12.9 \%$ $\pm 4 \%$. Almost two thirds of the patients (106 patients; $65 \%$ ) had abnormal LV-GLS. As expected, the mean LV-GLS in patients with an LVEF $<50 \%$ was significantly lower than that in patients with an LVEF $\geq 50 \%(-9.3 \%$ vs $-14.3 \%$, respectively; $P<.001$ ). However, even within the subgroup with LVEF $\geq 50 \%$, LV-GLS was abnormal in 54 $(45 \%)$ patients. The correlation between LVEF and LV-GLS was significant $(\mathrm{r}=-0.53 ; P<.001)$.

\section{Survival Analysis}

Over a mean follow-up of $6.6 \pm 4$ years, $88(54 \%)$ patients died. Mortality by 1 month occurred in only 4 $(2 \%)$ patients. However, an additional 12 patients $(8 \%)$ died in the first year. The cause of death was ascertained to be cardiopulmonary disease in $45(50 \%)$ patients and recurrent malignancy in $5(3 \%)$ patients. In the remaining 38 patients, cause of death could not be definitively determined. However, all patients were deemed to be free of malignancy at the time of their cardiac surgery.

The results of the univariable survival analysis are shown in Table E1 and indicate that lower LV-GLS, along with an increasing EuroSCORE, lack of a beta-blocker, and aspirin, was associated with increased long-term mortality. Other potential predictors (type of prior cancer, cardiac surgery, renal function, valvular regurgitation, LV volumes, and LVEF) were not significant predictors of mortality in the univariable analysis. Subsequently, stepwise, multivariable analysis was performed; the results are shown in Table 3. After adjustment for other variables, the association between LV-GLS and mortality became insignificant (HR 1.04 [95\% CI, 0.98-1.10]; $P=.2$ ), whereas EuroSCORE (HR 1.12 [95\% CI, 1.04-1.22]; $P=.002$ ), beta-blocker use (HR 0.67 [95\% CI, 0.47-0.96]; $P=.04$ ), and aspirin use (HR 0.66 [95\% CI, 0.44-0.98]; $P=.04$ ) remained significant. 


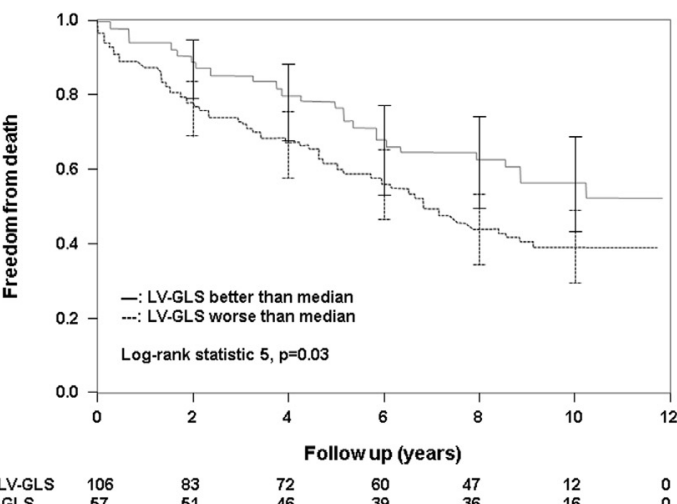

$\begin{array}{llllllll}\text { A Abnormal LV-GLS } & 106 & 83 & 72 & 60 & 47 & 12 & 0 \\ \text { Normal LV-GLS } & 57 & 51 & 46 & 39 & 36 & 16 & 0\end{array}$

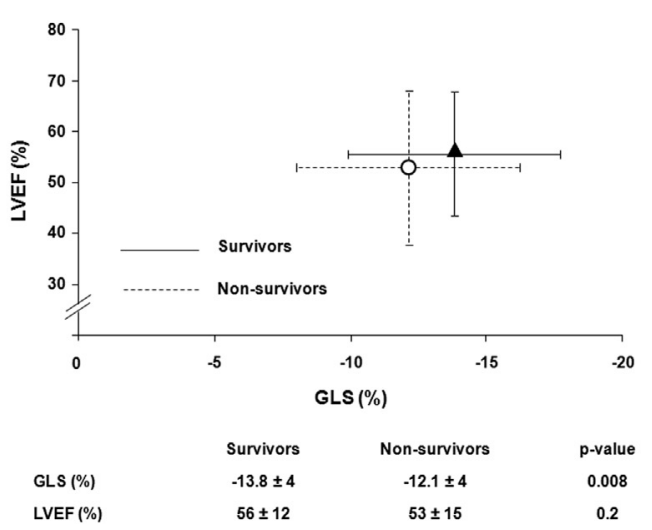

FIGURE 1. A, Kaplan-Meier survival curves of the entire study population, separated on the basis of preserved versus abnormal LV-GLS. B, LVEF and left ventricular GLS in survivors and nonsurvivors. LVEF, Left ventricular ejection fraction; $G L S$, global longitudinal strain.

For the entire study population, the mortality rate in patients with an abnormal LV-GLS (worse than $-14.5 \%$ ) was significantly higher than the rate in those with preserved LV-GLS (63 deaths in 106 patients [59\%] vs 25 deaths in 57 patients [44\%]; log-rank statistic 5; $P=.03$ ). The Kaplan-Meier survival curves of the entire study population, separated on the basis of preserved versus abnormal LV-GLS are shown in Figure 1, A. The findings were similar when only patients with preserved LVEF were included. Figure 1, $B$ demonstrates that the absolute mean LV-GLS was significantly higher in survivors versus nonsurvivors $(-13.8 \% \pm 4 \%$ vs $-12.1 \% \quad \pm 4 \%$; $P=.008)$, despite a similar LVEF $(56 \% \pm 12 \%$ vs $53 \pm$ $15 \% ; P=.2$ ). However, for both LVEF and LV-GLS, survivors and nonsurvivors showed a significant overlap.

To assess the discriminatory ability of LV-GLS to predict outcomes, we performed receiver operating characteristic analysis, using the data available at the 9-year cutoff, given that all patients had $\geq 9$ years of follow-up. The area under the curve was $0.62(P=.009)$. In addition, the HosmerLemeshow test was not significant $(P=.1)$, indicating that GLS and outcomes had a linear relationship.

To understand how the interplay between LVEF and LVGLS affected outcomes, we divided the patients into subgroups, based on whether they had preserved versus impaired LVEF (cutoff of 50\%) and preserved versus abnormal LV-GLS (cutoff of $-14.5 \%$ ). No patients had an impaired LVEF and a preserved LV-GLS. The proportions in each of the 3 remaining subgroups were significantly different (log-rank statistic $7 ; P=.04$ ): (1) preserved LVEF and preserved LV-GLS ( $\mathrm{n}=28$ of 65 ; $43 \%$ ); (2) preserved LVEF and abnormal LV-GLS $(\mathrm{n}=32$ of 54;59\%); and (3) impaired LVEF and abnormal LV-GLS ( $\mathrm{n}=28$ of $44 ; 64 \%$ ). The Kaplan-Meier curves demonstrating the outcomes of these 3 subgroups are shown in Figure E2, from which we inferred that the mortality in patients with impaired LVEF and abnormal LV-GLS is high, as would be expected. However, we additionally demonstrate that the mortality of the subgroup with

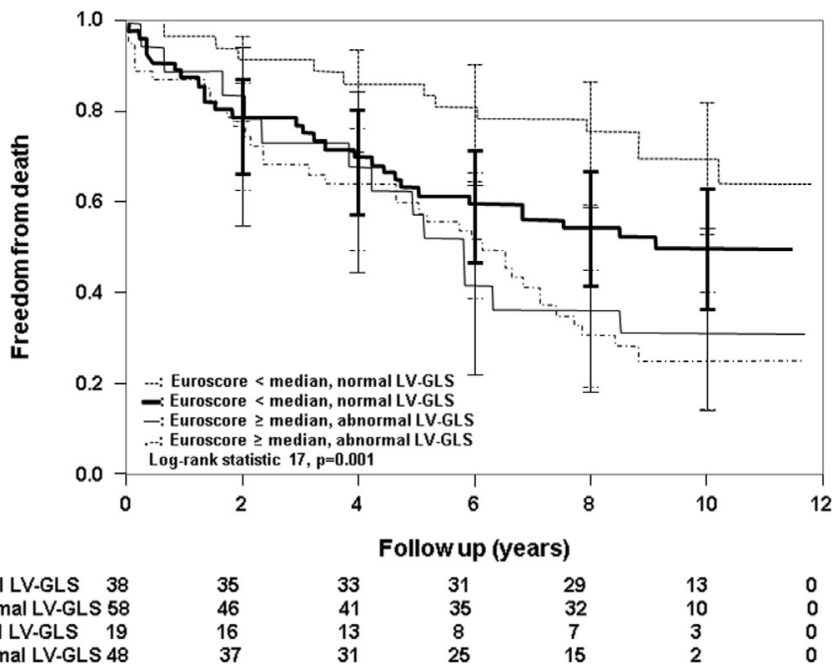

FIGURE 2. Kaplan-Meier survival curves, demonstrating the impact of EuroSCORE and LV-GLS on mortality. EuroSCORE, European System for Cardiac Operative Risk Evaluation; $L V$-GLS, left ventricular global longitudinal strain. 

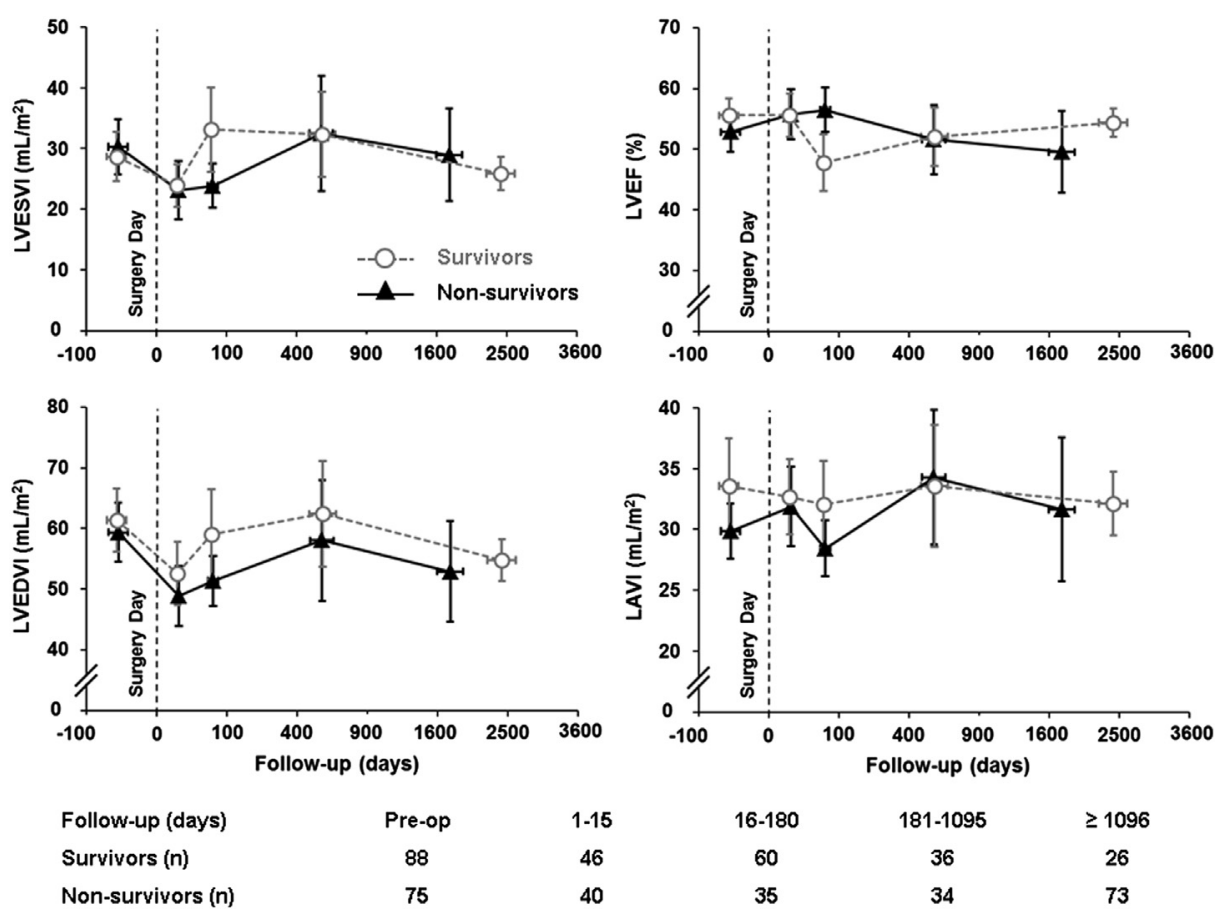

FIGURE 3. Temporal evolution of left ventricular volumetric parameters in survivors versus nonsurvivors, during follow-up. LVESVI, Left ventricular end-systolic volume index; $L V E F$, left ventricular ejection fraction; $L V E D V I$, left ventricular end-diastolic volume index; $L A V I$, left atrial volume index.

abnormal LV-GLS but preserved LVEF was significantly higher compared with those who had preserved LVEF and normal LV-GLS.

In addition, we divided the patients into 4 subgroups, based on whether they had a high versus low EuroSCORE (cutoff at a median of 8) and preserved versus abnormal LV-GLS (cutoff of $-14.5 \%$ ). The proportions in each of the 4 subgroups were as follows: (1) a low EuroSCORE and preserved LV-GLS ( $\mathrm{n}=12$ of $38 ; 32 \%$ ); (2) low EuroSCORE and abnormal LV-GLS $(\mathrm{n}=28$ of $58 ; 48 \%)$; and (3) high EuroSCORE and normal LV-GLS ( $\mathrm{n}=13$ of 19; $68 \%$ ); and (4) high EuroSCORE and abnormal LV-GLS $(\mathrm{n}=35$ of $48 ; 73 \%)$. The Kaplan-Meier curves demonstrating the outcomes of these 4 subgroups are shown in Figure 2 (log-rank statistic $17 ; P=.001)$. In patients with a high EuroSCORE, normal versus abnormal LV-GLS did not significantly affect mortality. However, in patients with a low EuroSCORE, having an abnormal LV-GLS was associated with significantly higher mortality, compared with those with normal LV-GLS.

\section{Longitudinal Data Analysis}

To understand whether the high mortality in these patients, observed over the long term of follow-up, was due to progressive decline in overt LV functional parameters, we subsequently studied the evolution of LV and left-atrial volumes and LVEF (obtained using echocardiography) over the course of the follow-up. As shown in Figure 3, during follow-up, no significant differences were found in LVEF, LV end-diastolic volume index, LV end-systolic volume index, or left-atrial volume index in survivors versus nonsurvivors. These results are indicative of the low utility of echocardiographic follow-up of parameters as a means to predict outcomes in patients with RACD.

\section{DISCUSSION}

We have previously shown that patients with RACD who undergo cardiothoracic surgery still experience a high rate of long-term mortality, compared with either a similaraged healthy population ( $86 \%$ survival at 10 years $)^{5}$ or an age- and gender-matched population undergoing similar types of cardiac surgery. ${ }^{16}$ The most frequent cause of death in this patient cohort was not recurrent malignancy but cardiorespiratory disease, despite surgical correction of underlying abnormalities. We therefore assessed whether these excess deaths can be explained by underlying myocardial dysfunction.

We demonstrate that standard echocardiographic parameters that reflect cardiac involvement, such as LVEF, severity of valvular disease, and right ventricle systolic pressure, did not predict outcomes in either univariable or multivariable analysis. On the other hand, the EuroSCORE, which is a cumulative score based on the combination of these parameters and clinical variables, was strongly predictive of mortality. This finding likely means that the degree of cardiac dysfunction is fairly advanced in patients with RACD and that mortality is better 
predicted by a combination of factors (eg, a composite EuroSCORE) than by individual predictors.

Parameters such as LVEF are recognized as lacking the level of sensitivity needed to detect early myocardial dysfunction. One possibility is that the current 2-dimensional techniques to measure LVEF by echocardiography (eg, Simpson's method), which typically do not function well with ischemic cardiomyopathy, could have affected the final results. Utilization of 3-dimensional techniques (eg, cardiac magnetic resonance or 3-dimensional echocardiography), which result in more-precise LVEF assessment, could potentially improve the discriminatory ability of LVEF to predict mortality in these patients. Another possibility is that in RACD patients with advanced valvular disease (eg, significant mitral regurgitation), what is commonly perceived as normal LVEF might actually be subnormal, thereby potentially affecting our results.

Finally, LV-GLS, a marker of regional myocardial dysfunction, is increasingly recognized as occurring earlier than overt LV systolic dysfunction. So the observation is not surprising that, despite having a normal LVEF, a sizable proportion of patients have an abnormal LV-GLS. In fact, medical decision making, based on assessment of LV-GLS, is 1 of the strategies being actively investigated in the field of cardio-oncology. Patients who receive chemotherapy are screened for development of abnormal LV-GLS, before onset of overt LV systolic dysfunction, so that medications such as beta-blockers can be administered to prevent progression to permanent LV dysfunction.

Hence, an intuitive hypothesis is that more-sensitive markers, such as LV-GLS, could potentially aid in prediction of mortality. However, in the current study, LV-GLS was a significant predictor of mortality in the univariable analysis; but it was not independently predictive in the multivariable analysis. This lack of independent predictive ability of LV-GLS for mortality in those with RACD is somewhat different from what has been described in other populations. Olsen and colleagues ${ }^{32}$ showed that reduced systolic strain, and LV strain rate, was associated with a poorer outcome in patients who have chronic severe aortic regurgitation, with either conservative treatment or aortic valve surgery. Recently, Dahl and colleagues ${ }^{33}$ demonstrated the long-term prognostic value of preoperative LV-GLS for cardiovascular mortality and morbidity after aortic valve replacement in patients with severe aortic stenosis with preserved LV contractility.

However, these cohorts were inherently different, and less ill, than the current study population. The deleterious effects of radiation on the heart are more diffuse than those in any other disease processes. ${ }^{7}$ Hence, patients with RACD could conceivably have a greater prevalence of extensive myocardial abnormalities, thus making a sensitive marker such as LV-GLS less discriminatory, from a prognostic perspective. However, in the study population, LV-GLS was more frequently abnormal, compared with standard measures of LV function, such as LVEF or LV volumes.

In addition, as shown in Figure 1, $B$, the mean LV-GLS was significantly worse in nonsurvivors, compared with survivors, despite similar LVEF, implying that it is a more sensitive marker in this population. As a result, LV-GLS, because it has higher sensitivity than LVEF, may have utility in the subgroup of patients with preserved LVEF. As shown in Figure E2, the mortality in the subgroup who had abnormal LV-GLS but preserved LVEF was significantly higher, compared with those who had preserved LVEF and normal LV-GLS. We additionally demonstrated that in higher-risk patients, as defined by a high EuroSCORE (ie, worse than a median score of 8), normal versus abnormal LV-GLS (based on a cutoff of $-14.5 \%$ ) did not have a significant impact on mortality. However, in lower-risk patients with a low EuroSCORE, having an abnormal LV-GLS was associated with significantly higher mortality, compared with those who had normal LV-GLS.

In the current study, we demonstrated that during the course of follow-up, the change in standard LV volumetric parameters (LVEF and indexed volumes) did not differ significantly in survivors versus nonsurvivors (Figure 3). This observation is important, as patients who have RACD continue to have a high rate of mortality after cardiac surgery. The current study demonstrates that the high mortality cannot be explained simply by deterioration in LV function or volumes. A more-sensitive marker, such as LV-GLS, potentially has a role, especially in those patients who have a preserved LVEF. In addition, we have to strive to find other potential predictors of poorer outcomes in this high-risk population. In a recent report, we demonstrated that RACD patients have a high proportion of pulmonary comorbidities (including pulmonary fibrosis), which prolongs recovery and independently contributes to increased mortality. ${ }^{34}$

\section{Clinical Implications}

Patients who have RACD are a challenging group; they have a heterogeneous presentation (coronary artery disease, valvular disease, cardiomyopathy, or pericardial disease) and often require complex cardiac surgeries. The rate of mortality is high and is not entirely explained by a progressive decline in global LV function. Assessment of LV-GLS, although it is more sensitive in determining regional LV dysfunction, does not add independent incremental utility (in addition to EuroSCORE) for predicting long-term outcomes. However, patients who have a preserved LVEF, but abnormal LV-GLS, are a higher-risk subgroup, meaning assessment of LV mechanics might provide incremental utility. Similarly, lower-risk patients (those with a low 
EuroSCORE but abnormal LV-GLS) are a higher-risk subgroup, meaning assessment of LV mechanics might provide incremental utility. In patients who have RACD, a comprehensive cardiopulmonary evaluation is necessary before they are referred for cardiac surgery. In patients that demonstrate high-risk features, alternate therapies, such as percutaneous coronary intervention and transcatheter valvular therapies, should be considered.

\section{Strengths and Limitations}

The current study had the following strengths and limitations. The study was large and observational, with potential bias resulting from tertiary-care referral; hence, the data are not generalizable to all patients with a prior history of thoracic radiation exposure. In addition, the type of cardiac pathology varied in these patients and included those with multivalvular abnormalities and advanced coronary disease. However, most patients who have RACD have multiple manifestations of their disease, reflecting the current reality of practice. Precise data on the total radiation dose and chemotherapeutic regimen, both of which could have an impact on outcomes, were not available in these patients. However, as is well known, radiation results in a different pattern of heart disease (proximal and/or ostial coronary artery disease, valvular disease, pericardial constriction, etc.), compared with chemotherapy-induced cardiotoxicity (LV dysfunction, abnormal myocardial mechanics, diastolic dysfunction, etc.).

In addition, the adverse effects from chemotherapy manifest much earlier than those from radiation (months vs years). We chose all-cause mortality because this measure is considered to be more objective and unbiased, compared with cardiac mortality. ${ }^{35,36}$ However, a majority of patients died from cardiopulmonary complications, and only a small proportion of patients had a documented recurrent malignancy during follow-up that resulted in death. In a sizable proportion of patients, cause of death could not be conclusively ascertained; however, all patients were deemed to be free of malignancy at the time of cardiac surgery.

\section{CONCLUSIONS}

Patients who have RACD have a high mortality rate after cardiac surgery, which is not independently predicted by standard echocardiographic parameters. Although LV-GLS is a sensitive marker of early systolic LV dysfunction, it does not sufficiently discriminate between survivors and nonsurvivors and is not independently predictive of long-term mortality in patients with RACD, beyond established markers such as the EuroSCORE. However, certain subgroups of patients with RACD (those who have a preserved LVEF but abnormal LV-GLS, and those with a low EuroSCORE but abnormal LVGLS) are at higher risk, meaning that assessment of LV mechanics might provide incremental utility. Future prospective studies are required to confirm these findings.

\section{Conflict of Interest Statement}

Authors have nothing to disclose with regard to commercial support.

\section{References}

1. Hancock SL, Tucker MA, Hoppe RT. Factors affecting late mortality from heart disease after treatment of Hodgkin's disease. JAMA. 1993;270:1949-55.

2. Handa N, McGregor CG, Danielson GK, Daly RC, Dearani JA, Mullany CJ, et al. Valvular heart operation in patients with previous mediastinal radiation therapy. Ann Thorac Surg. 2001;71:1880-4.

3. Handa N, McGregor CG, Danielson GK, Orszulak TA, Mullany CJ, Daly RC, et al. Coronary artery bypass grafting in patients with previous mediastinal radiation therapy. J Thorac Cardiovasc Surg. 1999;117:1136-42.

4. Nishimura RA, Carabello BA, Faxon DP, Freed MD, Lytle BW, O'Gara PT, et al. ACC/AHA 2008 guideline update on valvular heart disease: focused update on infective endocarditis: a report of the American College of Cardiology/American Heart Association Task Force on Practice Guidelines: endorsed by the Society of Cardiovascular Anesthesiologists, Society for Cardiovascular Angiography and Interventions, and Society of Thoracic Surgeons. Circulation. 2008;118: 887-96.

5. Chang AS, Smedira NG, Chang CL, Benavides MM, Myhre U, Feng J, et al. Cardiac surgery after mediastinal radiation: extent of exposure influences outcome. $J$ Thorac Cardiovasc Surg. 2007;133:404-13.

6. Glanzmann C, Kaufmann P, Jenni R, Hess OM, Huguenin P. Cardiac risk after mediastinal irradiation for Hodgkin's disease. Radiother Oncol. 1998;46:51-62.

7. Veinot JP, Edwards WD. Pathology of radiation-induced heart disease: a surgical and autopsy study of 27 cases. Hum Pathol. 1996;27:766-73.

8. Heidenreich PA, Hancock SL, Lee BK, Mariscal CS, Schnittger I. Asymptomatic cardiac disease following mediastinal irradiation. JAm Coll Cardiol. 2003;42:743-9.

9. Adams MJ, Lipshultz SE, Schwartz C, Fajardo LF, Coen V, Constine LS. Radiation-associated cardiovascular disease: manifestations and management. Semin Radiat Oncol. 2003;13:346-56.

10. Lee PJ, Mallik R. Cardiovascular effects of radiation therapy: practical approach to radiation therapy-induced heart disease. Cardiol Rev. 2005;13:80-6.

11. Arsenian MA. Cardiovascular sequelae of therapeutic thoracic radiation. Prog Cardiovasc Dis. 1991;33:299-311.

12. Crestanello JA, McGregor CG, Danielson GK, Daly RC, Dearani JA, Orszulak TA, et al. Mitral and tricuspid valve repair in patients with previous mediastinal radiation therapy. Ann Thorac Surg. 2004;78:826-31; discussion 826-31.

13. Reber D, Birnbaum DE, Tollenaere P. Heart diseases following mediastinal irradiation: surgical management. Eur J Cardiothorac Surg. 1995;9:202-5.

14. Veeragandham RS, Goldin MD. Surgical management of radiation-induced heart disease. Ann Thorac Surg. 1998;65:1014-9.

15. Hicks GL Jr. Coronary artery operation in radiation-associated atherosclerosis: long-term follow-up. Ann Thorac Surg. 1992;53:670-4.

16. Wu W, Masri A, Popovic ZB, Smedira NG, Lytle BW, Marwick TH, et al. Longterm survival of patients with radiation heart disease undergoing cardiac surgery: a cohort study. Circulation. 2013;127:1476-85.

17. Nahum J, Bensaid A, Dussault C, Macron L, Clemence D, Bouhemad B, et al. Impact of longitudinal myocardial deformation on the prognosis of chronic heart failure patients. Circ Cardiovasc Imaging. 2010;3:249-56.

18. Koyama J, Ray-Sequin PA, Falk RH. Longitudinal myocardial function assessed by tissue velocity, strain, and strain rate tissue Doppler echocardiography in patients with al (primary) cardiac amyloidosis. Circulation. 2003;107:2446-52.

19. Richand V, Lafitte S, Reant P, Serri K, Lafitte M, Brette S, et al. An ultrasound speckle tracking (two-dimensional strain) analysis of myocardial deformation in professional soccer players compared with healthy subjects and hypertrophic cardiomyopathy. Am J Cardiol. 2007;100:128-32.

20. Fuster V, Ryden LE, Cannom DS, Crijns HJ, Curtis AB, Ellenbogen KA, et al. ACC/AHA/ESC 2006 guidelines for the management of patients with atrial fibrillation: a report of the American College of Cardiology/American Heart Association Task Force on Practice Guidelines and the European Society of Cardiology Committee for Practice Guidelines (writing committee to revise the 2001 guidelines for the management of patients with atrial fibrillation): developed in collaboration with the European Heart Rhythm Association and the Heart Rhythm Society. Circulation. 2006;114:e257-354. 
21. Nashef SA, Roques F, Michel P, Gauducheau E, Lemeshow S, Salamon R. European system for cardiac operative risk evaluation (euroSCORE). Eur $J$ Cardiothorac Surg. 1999;16:9-13.

22. Lang RM, Bierig M, Devereux RB, Flachskampf FA, Foster E, Pellikka PA, et al, Recommendations for chamber quantification: a report from the American Society of Echocardiography's Guidelines and Standards Committee and the Chamber Quantification Writing Group, developed in conjunction with the European Association of Echocardiography, a branch of the European Society of Cardiology. J Am Soc Echocardiogr. 2005;18:1440-63.

23. Nagueh SF, Appleton CP, Gillebert TC, Marino PN, Oh JK, Smiseth OA, et al. Recommendations for the evaluation of left ventricular diastolic function by echocardiography. Eur J Echocardiogr. 2009;10:165-93.

24. Zoghbi WA, Enriquez-Sarano M, Foster E, Grayburn PA, Kraft CD, Levine RA, et al. Recommendations for evaluation of the severity of native valvular regurgitation with two-dimensional and Doppler echocardiography. J Am Soc Echocardiogr. 2003;16:777-802.

25. Bonow RO, Carabello BA, Kanu C, de Leon AC Jr, Faxon DP, Freed MD, et al. ACC/AHA 2006 guidelines for the management of patients with valvular heart disease: a report of the American College of Cardiology/American Heart Association Task Force on Practice Guidelines (writing committee to revise the 1998 guidelines for the management of patients with valvular heart disease): developed in collaboration with the Society of Cardiovascular Anesthesiologists: endorsed by the Society for Cardiovascular Angiography and Interventions and the Society of Thoracic Surgeons. Circulation. 2006;114:e84-231.

26. Wilkins GT, Weyman AE, Abascal VM, Block PC, Palacios IF. Percutaneous balloon dilatation of the mitral valve: an analysis of echocardiographic variables related to outcome and the mechanism of dilatation. Br Heart J. 1988;60:299-308.

27. Carasso S, Yang H, Woo A, Jamorski M, Wigle ED, Rakowski H. Diastolic myocardial mechanics in hypertrophic cardiomyopathy. J Am Soc Echocardiogr. 2010;23:164-71.
28. Carasso S, Yang H, Woo A, Vannan MA, Jamorski M, Wigle ED, et al. Systolic myocardial mechanics in hypertrophic cardiomyopathy: novel concepts and implications for clinical status. J Am Soc Echocardiogr. 2008;21:675-83.

29. Yang H, Carasso S, Woo A, Jamorski M, Nikonova A, Wigle ED, et al. Hypertrophy pattern and regional myocardial mechanics are related in septal and apical hypertrophic cardiomyopathy. J Am Soc Echocardiogr. 2010;23:1081-9.

30. Marwick TH, Leano RL, Brown J, Sun JP, Hoffmann R, Lysyansky P, et al. Myocardial strain measurement with 2-dimensional speckle-tracking echocardiography: definition of normal range. JACC Cardiovasc Imaging. 2009;2:80-4.

31. Fitzmaurice GM, Laird NM, Ware JH. Applied Longitudinal Analysis. Hoboken, NJ: Wiley; 2004.

32. Olsen NT, Sogaard P, Larsson HB, Goetze JP, Jons C, Mogelvang R, et al Speckle-tracking echocardiography for predicting outcome in chronic aortic regurgitation during conservative management and after surgery. JACC Cardiovasc Imaging. 2011;4:223-30.

33. Dahl JS, Videbaek L, Poulsen MK, Rudbaek TR, Pellikka PA, Moller JE. Global strain in severe aortic valve stenosis: relation to clinical outcome after aortic valve replacement. Circ Cardiovasc Imaging. 2012;5:613-20.

34. Desai MY, Karunakaravel K, Wu W, Agarwal S, Smedira NG, Lytle BW, et al. Pulmonary fibrosis on multidetector computed tomography and mortality in patients with radiation-associated cardiac disease undergoing cardiac surgery. $J$ Thorac Cardiovasc Surg. 2013;148:475-81.e3.

35. Boyle CA, Decoufle P. National sources of vital status information: extent of coverage and possible selectivity in reporting. Am J Epidemiol. 1990;131:160-8.

36. Lauer MS, Blackstone EH, Young JB, Topol EJ. Cause of death in clinical research: time for a reassessment? J Am Coll Cardiol. 1999;34:618-20.

Key Words: radiation-associated cardiac disease, echocardiography, strain, outcomes

\section{EDITORIAL COMMENTARY}

\section{A not-so-simple measure}

James I. Fann, MD

See related article on pages 1643-51.

One can picture a future in which a surgeon sitting comfortably at his console enters patient data into a computer that promptly generates the probability of surgical success. To a large extent today, the Society of Thoracic Surgeons National Database and the European System for Cardiac Operative Risk Evaluation (EuroScore) provide such assessments for many cardiac disorders. Further, clinicians currently rely on various diagnostic tests, many used for prognostication, to evaluate cardiac function during the

From the Department of Cardiothoracic Surgery, Stanford University Medical Center, Stanford, Calif.

Disclosures: Author has nothing to disclose with regard to commercial support.

Received for publication Feb 9, 2015; accepted for publication Feb 9, 2015; available ahead of print March 17, 2015.

Address for reprints: James I. Fann, MD, Department of Cardiothoracic Surgery, Stanford University Medical Center, FALK CVRC, 300 Pasteur Dr, Stanford, CA 94305 (E-mail: jfann@ @tanford.edu). perioperative period. However, there are diseases, such as those related to previous thoracic irradiation for malignancy, that continue to frustrate surgeons mainly because there are no good predictors of outcome. What is needed is a method to better understand the degree of myocardial

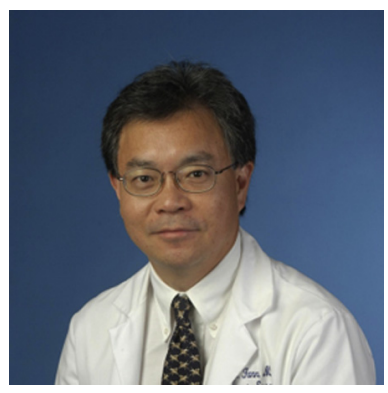
injury to have reliable estimates of recoverability in the short and long term. Not to be overlooked are noncardiacrelated confounders, such as the degree of pulmonary

J Thorac Cardiovasc Surg 2015;149:1651-2 0022-5223/\$0.00

Published by Elsevier Inc. on behalf of The American Association for Thoracic Surgery

http://dx.doi.org/10.1016/j.jtcvs.2015.02.018 

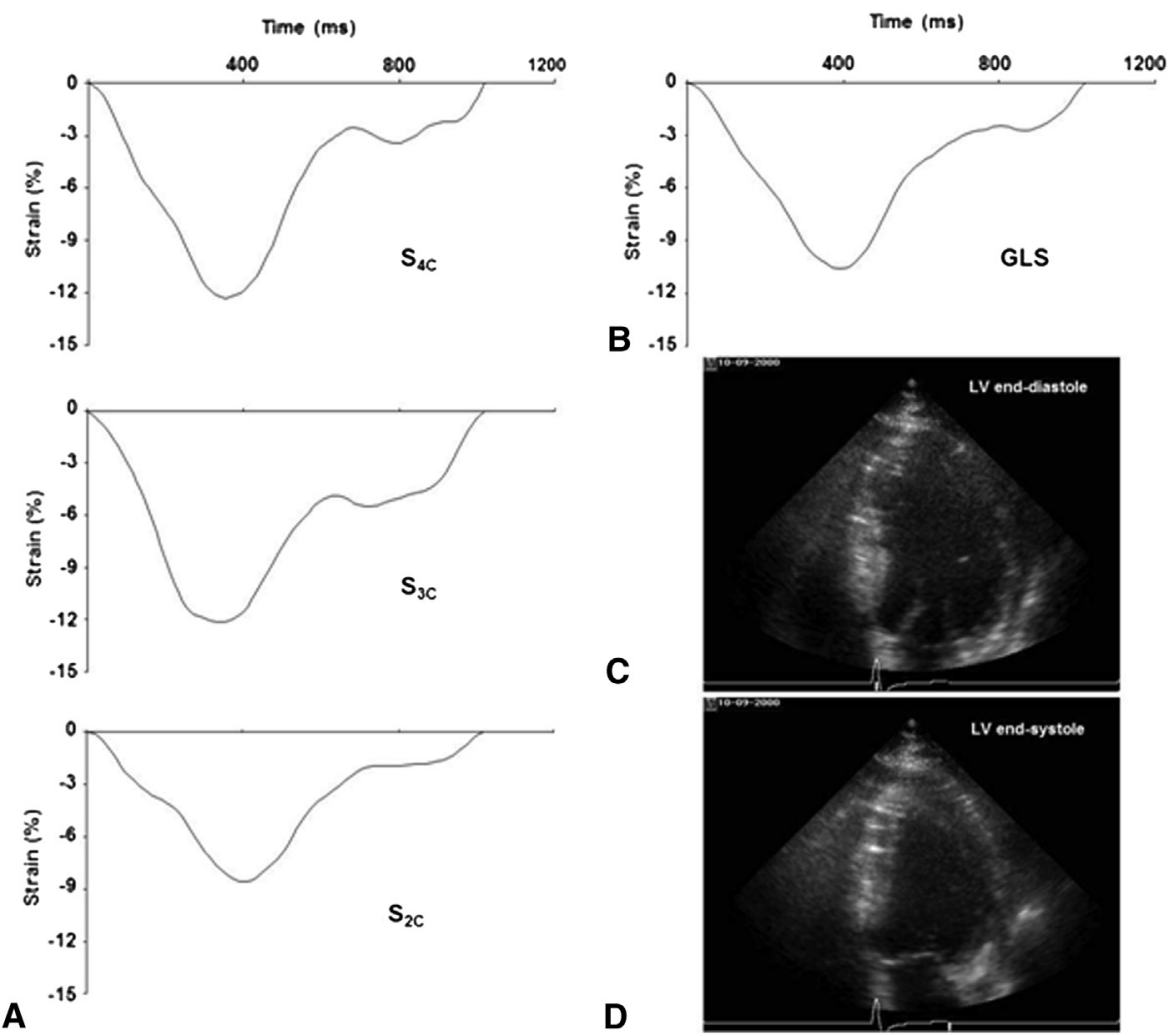

FIGURE E1. A, Average longitudinal strain curves derived from each apical view (A), and (B) average GLS. C and D, Two-dimensional images of left ventricle in apical 4-chamber view. $S_{4 C}$, Strain from 4-chamber; $G L S$, global longitudinal strain; $L V$, left ventricle; $S_{3 C}$, strain from 3-chamber; $S_{2 C}$, strain from 2-chamber.

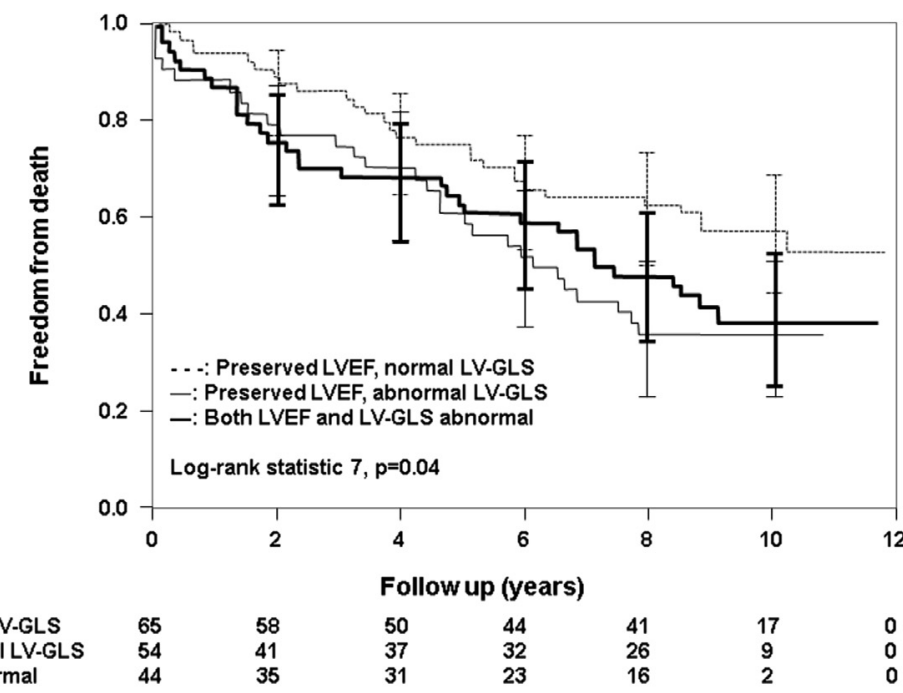

FIGURE E2. Kaplan-Meier survival curves, demonstrating the impact of LVEF and LV-GLS on mortality. LVEF, Left ventricular ejection fraction; $L V$ - $G L S$, left ventricular global longitudinal strain. 
TABLE E1. Univariable, Cox proportional hazard survival analysis for mortality in the study population

\begin{tabular}{lcl}
\hline \multicolumn{1}{c}{ Data } & Hazard & \\
& ratio (95\% CI) & $\boldsymbol{P}$ \\
\hline Preoperative & & \\
Age & $1.02(1.001-1.03)$ & .03 \\
Gender & $0.64(0.40-1.03)$ & .05 \\
Hypertension & $0.96(0.64-1.45)$ & .4 \\
Diabetes Mellitus & $0.66(0.91-2.51)$ & .08 \\
Hyperlipidemia & $0.79(0.51-1.20)$ & .2 \\
Prior stroke & $1.09(0.63-1.87)$ & .8 \\
Proximal obstructive coronary artery disease & $1.14(0.75-1.72)$ & .5 \\
Atrial fibrillation & $1.05(0.62-1.78)$ & .8 \\
Implantable cardioverter defibrillator & $1.18(0.47-2.98)$ & .8 \\
Permanent pacemaker & $1.43(0.77-2.66)$ & .3 \\
Type of prior cancer & $1.03(0.89-1.20)$ & .7 \\
Prior heart surgery & $1.07(0.91-1.26)$ & .3 \\
Type of cardiac surgery performed & $1.09(0.93-1.26)$ & .3 \\
EuroSCORE & $1.14(1.07-1.22)$ & $<.001$ \\
Beta-blockers & $0.51(0.33-0.77)$ & .002 \\
Angiotensin-converting enzyme inhibitors & $0.90(0.56-1.42)$ & .2 \\
Aspirin & $0.56(0.37-0.84)$ & .005 \\
Clopidogrel & $1.07(0.27-4.17)$ & .9 \\
Statins & $0.95(0.60-1.52)$ & .4 \\
Serum creatinine & $1.49(1.03-2.23)$ & .02 \\
Left ventricular ejection fraction & $0.99(0.98-1.005)$ & .3 \\
Left ventricular end-systolic volume index & $0.98(0.95-1.02)$ & .3 \\
Left atrial volume index & $0.99(0.98-1.004)$ & .2 \\
Mitral regurgitation & $0.97(0.82-1.15)$ & .7 \\
Aortic regurgitation & $1.18(0.95-1.47)$ & .13 \\
Right ventricular systolic pressure & $1.007(0.99-1.02)$ & .3 \\
Diastolic dysfunction & $1.04(0.83-1.30)$ & .8 \\
Mitral valve splitability score & $0.99(0.93-1.06)$ & .9 \\
Aortic valve dimensionless index & $0.54(0.19-1.55)$ & .26 \\
Left ventricular global longitudinal strain & $1.07(1.01-1.14)$ & .006 \\
Postoperative & & \\
Left ventricular ejection fraction & $0.99(0.98-1.01)$ & .8 \\
Mitral regurgitation & $1.04(0.77-1.40)$ & .8 \\
Aortic regurgitation & $1.20(0.70-2.05)$ & .5 \\
Right ventricular systolic pressure & $1.02(0.98-1.05)$ & .3 \\
Right ventricular dysfunction & $0.94(0.60-1.47)$ & .8 \\
Permanent atrial fibrillation & $0.61(0.30-1.25)$ & .12 \\
\hline EI, Confidence interval; EuroSCORE, European System for Cardiac Operative Risk \\
Evaluation. & & \\
& &
\end{tabular}

